\title{
Flebotomíneos (Diptera, Psychodidae) de área de transmissão de leishmaniose tegumentar americana, no município de Itupeva, região sudeste do Estado de São Paulo, Brasil
}

\author{
Sand flies (Diptera, Psychodidae, Phlebotominae) of the American \\ cutaneous leishmaniasis transmition area in the southeastern \\ region of the São Paulo State, Brazil

\begin{abstract}
Renata Caporalle Mayo, Claudio Cosanova, Luciene Maura Mascarini, Dalva Marli Valério Wanderley e Fernando Motta de Azevedo Corrên
\end{abstract} \\ Marta Gislene Pignatti, Osias Rangel, Eunice Aparecida Bianchi Galati,
}

\begin{abstract}
Resumo O estudo da fauna flebotomínica em área de transmissão recente de leishmaniose tegumentar americana fornece subsídios para o controle da doença no Estado de São Paulo. Neste estudo, procurou-se caracterizar a distribuição sazonal, espacial e horária das espécies de flebotomíneos encontradas no município de Itupeva. As coletas foram realizadas quinzenalmente entre abril/94 e março/95. Dos 864 indivíduos coletados, 81,4\% pertenceram a 4 espécies: Lutzomyia migonei (32,4\%), L. whitmani (26,0\%), L. intermedia $(12,0 \%)$ e L. fischeri $(10,9 \%)$. Estas espécies apresentaram maior densidade no período mais frio e seco do ano (abril a setembro/94) e maior atividade entre a segunda e a quinta hora após o crepúsculo vespertino. L. migonei predominou em praticamente todos os ambientes investigados, seguida por L. whitmani e L. longipalpis no ambiente domiciliar. Conclui-se que, juntamente com L. intermedia, espécie suspeita como provável vetora no Estado de São Paulo, L. migonei e L. whitmani podem estar desempenhando um importante papel na transmissão da leishmaniose tegumentar na área estudada.
\end{abstract}

Palavras-chaves: Phlebotominae. Fauna flebotomínica. Ecologia de vetores. Leishmaniose tegumentar americana.

\begin{abstract}
The present study was effected aiming the verification of the seazonal, spatial and hourly of the phlebotominic species found in the county of Itupeva. Captures were performed fortnightly and 864 sand flies were collected between april/94 and march/95. 81.3\% of the captured specimens belonged to 4 species: L. migonei (32.4\%), L. whitmani (26.0\%), L. intermedia (12.0\%) e L. fischeri (10.9\%). Such species showed larger densities during the cold and dry season of the year (from april to september/94) and were more active between the second and the fifth hour after twilight. L. migonei predominated almost in all the investigated surroundings being followed by L. whitmani and L. Iongipalpis in the domestic environnents. In conclusion it is thought that in conjunction with $\mathrm{L}$. intermedia, a suspect vector in the State of São Paulo, L. migonei and L. whitmani may have an important role in the transmission of cutaneous leishmaniasis in the area under study.
\end{abstract}

Key-words: Phlebotominae. Phlebotominic fauna. Vector ecology. American cutaneous leishmaniasis.

Superintendência de Controle de Endemias (SUCEN) e Faculdade de Saúde Pública da Universidade de São Paulo. Auxílio Financeiro PCDEN e SUCEN

Endereço para correspondência: Dr. Claudio Casanova. SUCEN. R. Afonso Pessini 86, 13840-970 Mogi-Guaçu, SP, Brasil.

Recebido para publicação em 17/09/97. 
A leishmaniose tegumentar americana (LTA), no Estado de São Paulo, apresentou-se no passado com aspecto geográfico localizado e alta incidência entre os trabalhadores que exerciam atividades relacionadas às florestas 9 . Com o passar de várias décadas, este quadro foi se alterando, apresentando, a partir dos anos 70, uma nova feição epidemiológica, na qual a endemicidade e a ocorrência de casos esporádicos e/ou micro surtos caracterizam o padrão atual da transmissão de LTA no território paulista36 1213.

Micro surtos ocorridos recentemente em municípios que nunca haviam notificado casos de leishmaniose ou, que pelo menos não o faziam há muito tempo, têm contribuído de modo importante para o perceptível aumento do número de casos autóctones e da área de distribuição da LTA no Estado de São Paulo a partir de 1990 (CVE - Secretaria da Saúde do

\section{Estado de São Paulo).}

Embora, Lutzomyia intermedia seja reconhecida como provável vetor em diversas regiões do Estado, consideradas como endêmicas2 46711 , pouco se conhece sobre a fauna e o comportamento das espécies de flebotomíneos em área de recente transmissão da leishmaniose tegumentar.

Este estudo teve por objetivo caracterizar a fauna e a distribuição sazonal, espacial e horária das espécies de flebotomíneos em uma localidade rural, no Bairro Quilombo, do município de Itupeva, o qual apresentou seus primeiros 12 casos de leishmaniose tegumentar no ano de 199210.

\section{MATERIAL E MÉTODOS}

Área de estudo. O estudo foi realizado na Fazenda Capim Fino, situada no Bairro Quilombo,
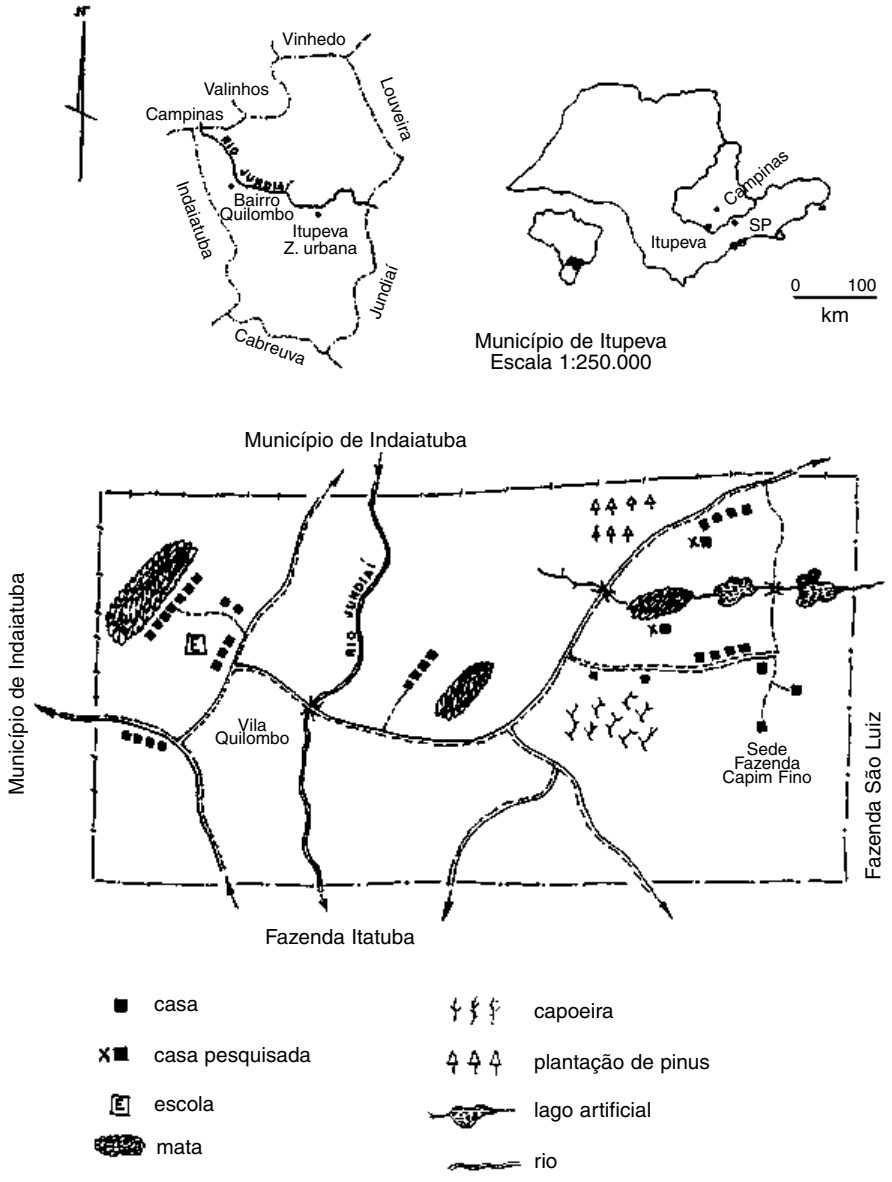

Figura 1 - Localização geográfica do bairro Quilombo, onde situa-se a fazenda Capim Fino, no município de Itupeva, Săo Paulo, Brasil. 
a aproximadamente $10 \mathrm{~km}$ do Centro Urbano de

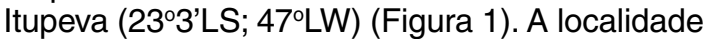
está situada próximo ao rio Jundiaí, pertencente à bacia do rio Tietê. Apresenta morros com afloramentos rochosos e áreas de vegetação natural, caracterizada como floresta mesófila semidecídua, e de reflorestamentos.

O clima predominante da região é do tipo mesotérmico de inverno seco (CWB), apresentando um período quente e úmido, correspondente aos meses de outubro a março e um período frio e seco, entre abril e setembro.

Metodologia. Foram realizadas coletas quinzenais de formas adultas de flebotomíneos no ambiente domiciliar e de mata, no período de abril/94 a março/95.

Os métodos de captura dos flebotomíneos foram armadilhas luminosas (tipo CDC), distribuídas no intra e peridomicílio, na margem e no interior da mata, e armadilha de Shannon, instalada entre a mata e as habitações humanas.

Os horários das capturas foram diferenciados conforme a armadilha empregada. As CDCs foram instaladas do crepúsculo vespertino ao matutino e, com exceção daquelas do intra domicílio, todas tiveram os coletores trocados no meio deste período. A armadilha de Shannon funcionou por um período de 6 horas a partir do crepúsculo vespertino, sendo que, em cada estação do ano foi realizada uma coleta de 12 horas.

\section{RESULTADOS}

A fauna flebotomínica da Fazenda Capim Fino constitui-se de 14 espécies: Lutzomyia migonei (França, 1920), L. Whitmani (Antunes \&
Coutinho, 1939), L. intermedia (Lutz \& Neiva, 1912), L. fischeri (Pinto, 1926), L. longipalpis (Lutz \& Neiva, 1912), L. firmatoi (Barretto, Martins \& Pellegrino, 1956), L. pessoai (Coutinho \& Barretto, 1940), L. alphabetica (Fonseca, 1936), L. monticola (Costa Lima, 1932), L. borgmeieri (Martins, Falcão \& Silva, 1972), L. Ienti (Mangabeira, 1938), L. cortelezzii (Brèthes, 1923), L. shannoni (Dyar, 1929) e Brumptomyia sp.

$\mathrm{Na}$ Tabela 1, está registrado o total geral de flebotomíneos coletados em todas as armadilhas e ambientes pesquisados. Apesar da grande diversidade de espécies coletadas, $81,4 \%$ dos flebotomíneos pertencem a 4 espécies: L. migonei (32,4\%), L. whitmani $(26,0 \%)$, L. intermedia $(12,0 \%)$ e L. fischeri $(10,9 \%)$.

A Figura 2 apresenta a variação sazonal das quatro espécies mais abundantes, coletadas em armadilha de Shannon e a Figura 3, a flutuação de alguns parâmetros metereológicos durante o período de estudo. Houve uma tendência de sazonalidade nas 4 espécies, com picos de densidade registrados durante o período mais frio e seco do ano (abril a setembro/94) e baixas densidades durante o período mais quente e úmido (outubro a março/95).

A distribuição horária das quatro espécies mais abundantes pode ser observada através das coletas realizadas em armadilha de Shannon (Figura 4). Estas espécies apresentaram maior atividade entre a segunda e a quinta hora após o crepúsculo vespertino, atingindo um pico na terceira hora. As coletas realizadas durante períodos de 12 horas não possibilitaram análises comparativas entre o primeiro e o segundo turno, devido ao número reduzido de indivíduos coletados nestas ocasiões.

$\mathrm{Na}$ Tabela 2, são apresentados os resultados

Tabela 1 - Flebotomíneos coletados no município de Itupeva, Estado de São Paulo, Brasil, no período de abril/94 a março/95.

\begin{tabular}{|c|c|c|c|c|}
\hline Espécies & Machos & Fêmeas & Total & $\%$ \\
\hline L. migonei & 141 & 139 & 280 & 32,4 \\
\hline L. whitmani & 83 & 142 & 225 & 26,0 \\
\hline L. intermedia & 40 & 64 & 104 & 12,0 \\
\hline L. fischeri & 40 & 54 & 94 & 10,9 \\
\hline L. longipalpis & 33 & 27 & 60 & 6,9 \\
\hline L. firmatoi & 6 & 25 & 31 & 3,6 \\
\hline L. pessoai & 11 & 11 & 22 & 2,5 \\
\hline L. alphabetica & 1 & 13 & 14 & 1,6 \\
\hline L. monticola & 0 & 14 & 14 & 1,6 \\
\hline L. borgmeieri & 5 & 7 & 12 & 1,4 \\
\hline L. lenti & 0 & 2 & 2 & 0,2 \\
\hline L. cortelezzii & 0 & 2 & 2 & 0,2 \\
\hline L. shannoni & 1 & 1 & 2 & 0,2 \\
\hline Brumptomyia sp & 1 & 1 & 2 & 0,2 \\
\hline Total & 362 & 502 & 864 & 100,0 \\
\hline
\end{tabular}




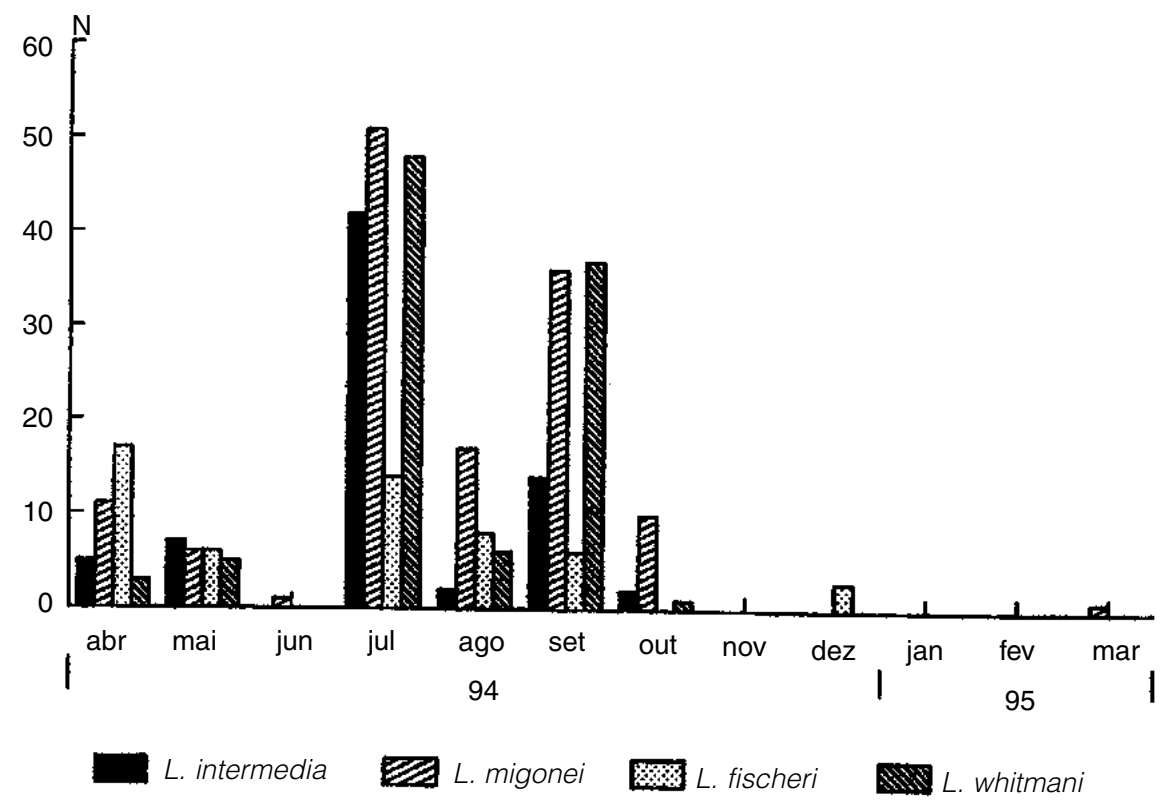

Figura 2 - Distribuição sazonal das espécies de flebotomíneos mais freqüentes coletados em armadilha de Shannon no município de Itupeva, Estado de São Paulo, Brasil, no período de abril/94 a março/95 ( $N=$ número de indivíduos).

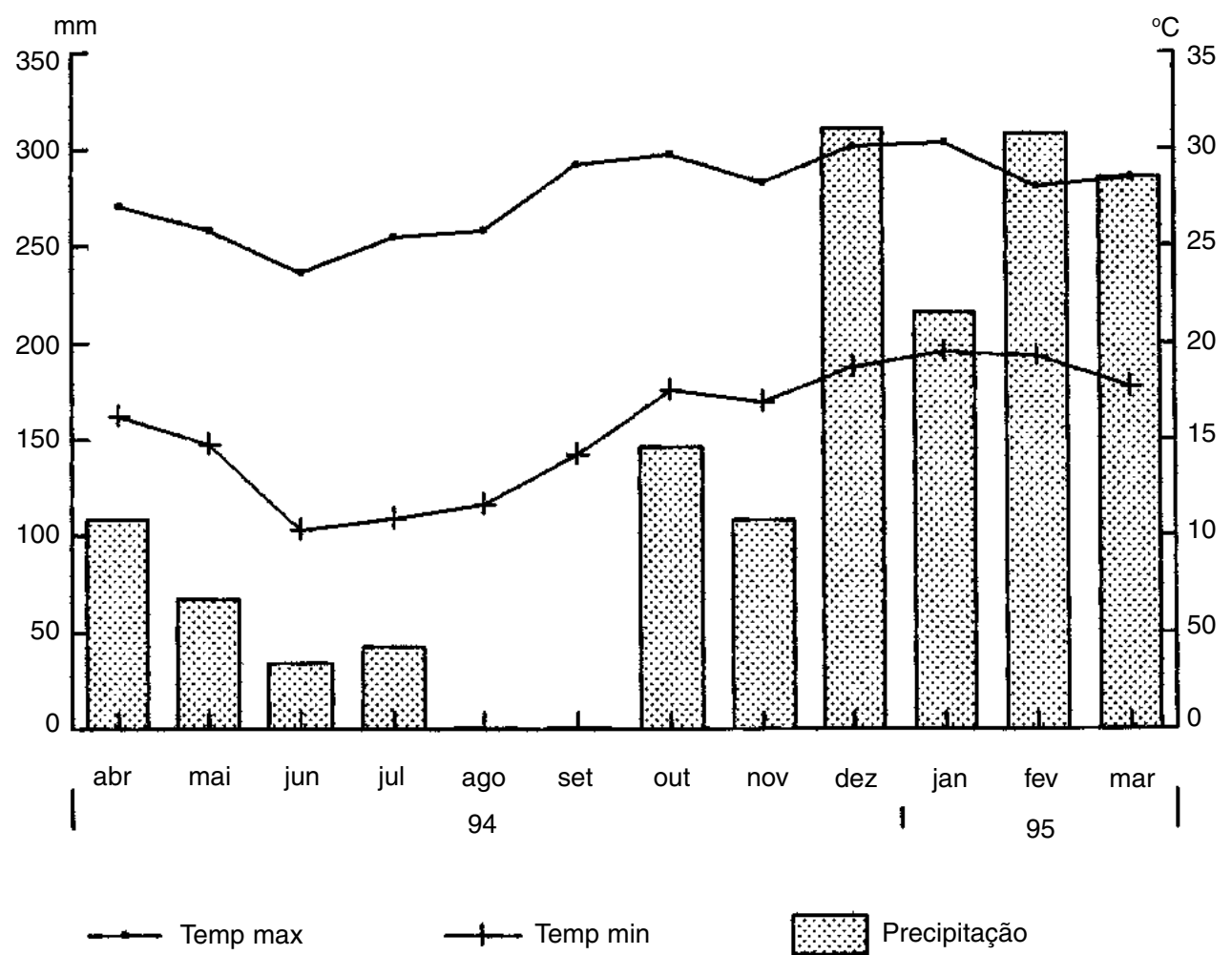

Figura 3 - Distribuição mensal da precipitação e das médias das temperaturas máximas e mínimas no município de Itupeva, Estado de São Paulo, Brasil, no período de abril/94 a março/95. 


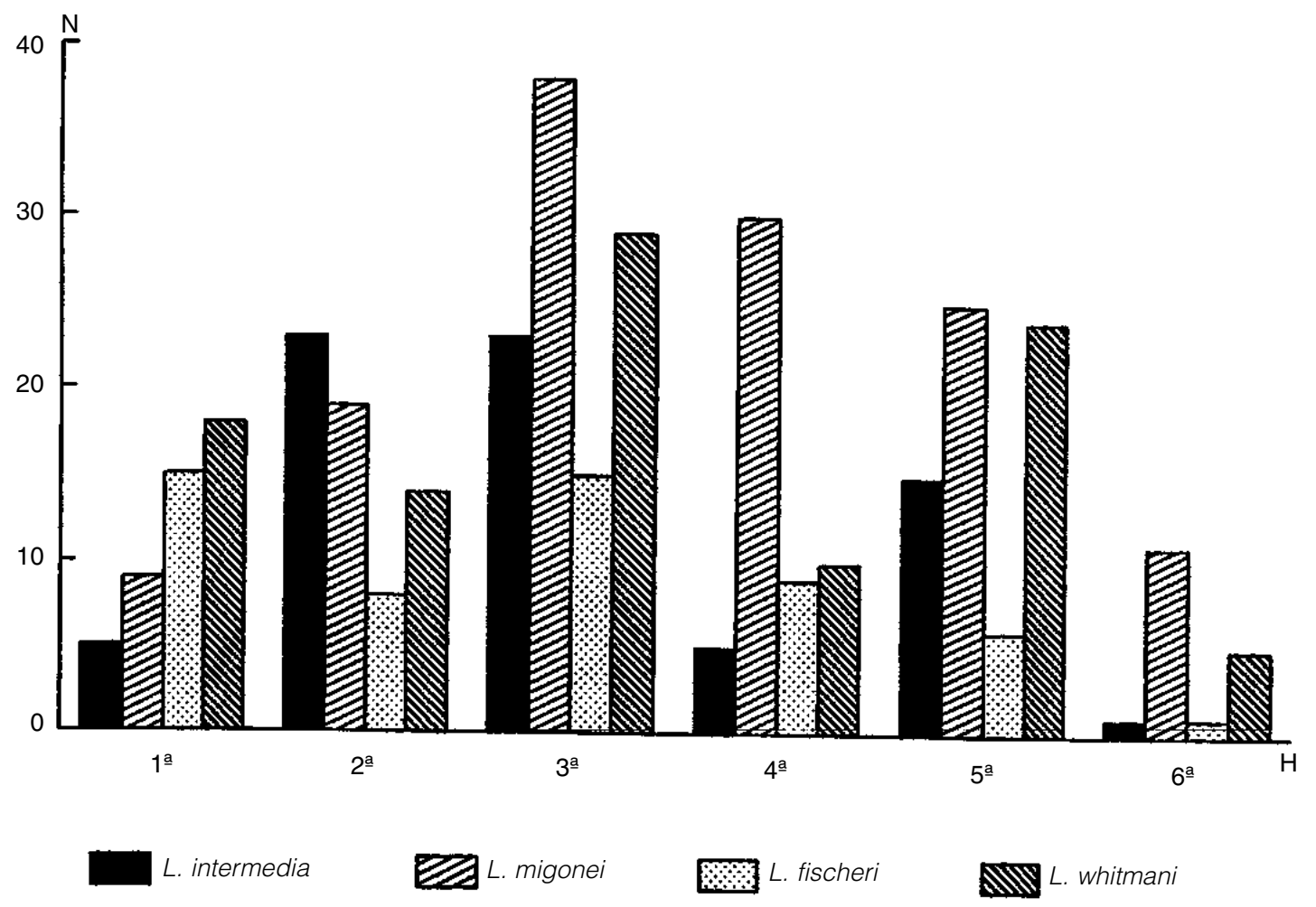

Figura 4 - Distribuição horária de flebotomíneos mais freqüentes coletados em armadilhas de Shannon no município

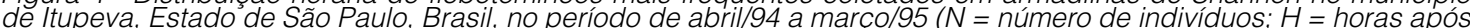
crepúsculo vespertino).

Tabela 2 - Freqüência de flebotomíneos coletados em armadilhas CDC, segundo ambiente, no município de Itupeva, Estado de São Paulo, Brasil, no período de abril/94 a março/95.

\begin{tabular}{|c|c|c|c|c|c|c|c|c|c|c|c|c|c|c|c|}
\hline \multirow{4}{*}{ Espécies } & \multicolumn{8}{|c|}{ Ambiente domiciliar } & \multirow{2}{*}{\multicolumn{4}{|c|}{ Ambiente florestal }} & & & \multirow{4}{*}{ Total } \\
\hline & \multicolumn{4}{|c|}{ intra } & \multicolumn{4}{|c|}{ peri } & & & & & \multirow{2}{*}{\multicolumn{2}{|c|}{ Subtotal }} & \\
\hline & \multicolumn{2}{|c|}{ casa 1} & \multicolumn{2}{|c|}{ casa 2} & \multicolumn{2}{|c|}{ casa 1} & \multicolumn{2}{|c|}{ casa 2} & \multicolumn{2}{|c|}{ margem } & \multicolumn{2}{|c|}{ interior } & & & \\
\hline & $\mathrm{m}$ & $f$ & $\mathrm{~m}$ & $f$ & $\mathrm{~m}$ & $f$ & $\mathrm{~m}$ & $f$ & $\mathrm{~m}$ & $f$ & $\mathrm{~m}$ & $f$ & $\mathrm{~m}$ & $f$ & \\
\hline L. migonei & - & 4 & 9 & 17 & 37 & 22 & 24 & 23 & - & 1 & 8 & 1 & 78 & 68 & 146 \\
\hline L. whitmani & 1 & 4 & 1 & 4 & 16 & 42 & 36 & 22 & - & - & - & 1 & 54 & 73 & 127 \\
\hline L. longipalpis & - & 1 & 1 & 2 & 26 & 18 & 3 & 5 & - & - & - & 1 & 30 & 27 & 57 \\
\hline L. fischeri & 2 & 1 & 2 & 3 & 4 & 12 & 7 & 9 & - & - & - & 2 & 15 & 27 & 42 \\
\hline L. intermedia & 1 & - & - & 1 & 5 & 4 & 11 & - & - & - & 3 & 4 & 20 & 9 & 29 \\
\hline L. pessoai & - & - & - & - & 4 & 1 & 3 & 1 & - & - & - & - & 7 & 2 & 9 \\
\hline L. borgmeieri & 3 & 1 & 1 & - & - & 1 & - & - & - & - & - & 1 & 4 & 3 & 7 \\
\hline L. alphabetica & - & 1 & - & - & - & 1 & - & - & - & - & - & 6 & - & 8 & 8 \\
\hline L. monticola & - & - & - & - & - & 1 & - & 1 & - & - & - & 3 & - & 5 & 5 \\
\hline L. firmatoi & 4 & 1 & - & 2 & - & - & 2 & - & - & - & - & - & 6 & 3 & 9 \\
\hline L. cortelezzii & - & - & - & - & - & 1 & - & - & - & - & - & - & - & 1 & 1 \\
\hline L. shannoni & - & - & - & - & - & - & - & - & - & - & - & 1 & - & 1 & 1 \\
\hline L. lenti & - & 1 & - & 1 & - & - & - & - & - & - & - & - & - & 2 & 2 \\
\hline Brumptomyia sp & - & - & - & - & - & - & - & - & - & - & - & 1 & - & 1 & 1 \\
\hline Total & 11 & 14 & 14 & 30 & 92 & 103 & 86 & 61 & - & 1 & 11 & 21 & 214 & 230 & 444 \\
\hline
\end{tabular}

$\mathrm{m}=$ macho; $\mathrm{f}$ = fêmea. 
relativos às freqüências de flebotomíneos coletados nas armadilhas luminosas CDC, distribuídas de acordo com o ambiente investigado. Embora com freqüências relativas bem diferenciadas, a maioria das espécies compareceram em todos os ambientes, excetuando o da margem da mata. $L$. migonei apresentou-se como a espécie dominante em praticamente todos os ambientes, seguido por L. whitmani e L. longipalpis no ambiente domiciliar.

\section{DISCUSSÃO}

A presença de 14 espécies de flebotomíneos indica uma alta diversidade na área de estudo, provavelmente, em virtude da vegetação e relevo diversificados. Investigações efetuadas em outras regiões de planalto do Estado de São Paulo, demonstraram que apenas algumas espécies, tais como $L$. whitmani, L. intermedia, L. migonei, L. fischerie $L$. pessoai apresentaram-se como sendo mais abundantes em ambientes antrópicos, incluindo o domicílio humano4 9 11. Em tais investigações, ora uma, ora outra parecem ter destacado papel na transmissão de LTA, haja vista as características que lhes conferem capacidade vetorial. Apesar da diversidade, a predominância quase absoluta de poucas espécies (Tabela 1) coincide com dados de outros autores 1511.

O predomínio de fêmeas (Tabela 1) não constitui achado novo e pode ser explicado pela atração exercida tanto pela luz como pela disponibilidade de fonte sanguínea próxima às armadilhas5.

A variação sazonal apresentada por L. migonei, L. whitmani, L. intermedia e L. fischeri (Figura 2) parece indicar que o período mais seco do ano deve favorecer o desenvolvimento dessas espécies (Figura 3). A queda brusca no número de flebotomíneos observada no mês de junho/94, possivelmente esteve relacionada aos baixos índices de temperatura e à ocorrência de ventos moderados nos dias em que foram realizadas as coletas, fatores já reconhecidos como interferentes na atividade dos flebotomíneos e no rendimento das armadilhas utilizadas 1 . No entanto, a ocorrência de intensas queimadas na área de estudo, no mês de outubro, às quais provocaram acentuada modificação ambiental, deve ser levada em conta como um possível fator de interferência na dinâmica populacional das espécies, a partir deste evento.
Levando-se em conta os horários de maior atividade das 4 espécies mais abundantes (Figura 4), pode-se concluir que o intervalo compreendido entre a segunda e a quinta hora após o crepúsculo vespertino, representaria o período de maior risco de infecção para a população local.

A ocorrência de praticamente as mesmas espécies, tanto no ambiente domiciliar como no de mata (Tabela 2), deve-se, possivelmente, à proximidade dos mesmos. A adaptação de certas espécies de flebotomíneos ao ambiente modificado pelo homem tem sido responsabilizada pela modificação do perfil epidemiológico da LTA no Estado de São Paulo6 7812 13. Nesse sentido, a predominância de $L$. migonei e $L$. whitmani no ambiente domiciliar indica que estas espécies, juntamente com L. intermedia, podem desempenhar importante papel na transmissão da leishmaniose tegumentar na área em estudo. A ausência de praticamente todas as espécies na armadilha luminosa instalada na margem da mata, pode estar relacionada à maior exposição deste ambiente aos ventos moderados ou fortes que ocorreram em alguns dias de coleta.

A presença de $L$. longipalpis, quase que exclusivamente no ambiente domiciliar, revestese de importância para a Vigilância Epidemiológica devido ao risco de introdução de leishmaniose visceral no Estado de São Paulo.

\section{REFERÊNCIAS BIBLIOGRÁFICAS}

1. Aguiar GM, Vilela ML, Schuback PA, Soucaux T, Azevedo ACR. Aspectos da ecologia dos flebótomos do Parque Nacional da Serra dos orgãos, Rio de Janeiro. IV - Freqüência mensal em armadilhas luminosas (Diptera, Psychodidae, Phlebotominae). Memórias do Instituto Oswaldo Cruz 80:465-82, 1985.

2. Forattini OP, Rabelo EX, Serra OP, Galati EAB, Barata JMS. Observações sobre a transmissão da leishmaniose tegumentar no Estado de São Paulo, Brasil. Revista de Saúde Pública 10:31-43, 1976.

3. Gomes AC. Perfil Epidemiológico da leishmaniose tegumentar no Brasil. Anais Brasileiros de Dermatologia 67:55-60, 1992.

4. Gomes AC, Barata JMS, Rocha, Silva EO, Galati EAB. Aspectos ecológicos da leishmaniose tegumentar americana. 6. Fauna flebotomínea antropófila de matas residuais situadas na região 
Centro-Nordeste do Estado de São Paulo, Brasil. Revista do Instituto de Medicina Tropical 31:32-39, 1989.

5. Gomes AC, Galati EAB. Aspectos ecológicos da leishmaniose tegumentar americana. 5. Estratificação da atividade espacial e estacional de Phlebotominae (Diptera, Psychodidae) em áreas de cultura agrícola da região do Vale do Ribeira, Estado de São Paulo, Brasil. Memórias do Instituto Oswaldo Cruz 82:46773, 1987.

6. Gomes AC, Rabello EX, Santos JLF, Galati EAB. Aspectos da leishmaniose tegumentar americana. 1. Estudo experimental da freqüência de flebotomíneos a ecótopos artificiais com referência especial a Psychodopygus intermedius. Revista de Saúde Pública 14:540-56, 1980.

7. Gomes AC, Santos JLF, Galati EAB. Ecological aspects of American cutaneous leishmaniasis. 4. Observations on the endophilic behavior of the sandfly and the vectorial role of Psychodopygus intermedius in the Ribeira Valley region of the São Paulo State, Brazil. Revista de Saúde Pública 20:28087, 1986.

8. Marzochi, MCA, Marzochi, KBF. Tegumentary and visceral leishmaniases in Brazil - Emerging anthropozoonosis and possibilities for their control. Cadernos de Saúde Pública 10:359-375, 1994.

9. Pessôa SB, Barretto MP. Leishmaniose tegumentar americana. Imprensa Nacional, Rio de Janeiro, Brasil, 1948.

10. Pignatti MG, Mayo RC, Alves MJCP, Souza SSAL, Macedo F, Pereira RM. Leishmaniose tegumentar americana na região nordeste do Estado de São Paulo - Brasil. Revista da Sociedade Brasileira de Medicina Tropical 28:243-47, 1995.

11. Taniguchi HH, Tolezano JE, Corrêa FMA, Moraes RHP, Marasa AM. Epidemiologia da leishmaniose tegumentar americana no Estado de São Paulo, Brasil. I. Composição da fauna flebotomínica no Município de São Roque, região de Sorocaba. Revista do Instituto Adolfo Lutz 51:23-30, 1991.

12. Tolezano JE. Epidemiological aspects of american cutaneous leishmaniasis en the State of São Paulo, Brasil. Memórias do Instituto Oswaldo Cruz 89:427434, 1994.

13. Tolezano JE, Marcoris SAG, Diniz JMP. Modificação na epidemiologia da leishmaniose tegumentar na 